\title{
DISCLAIMER
}

This report was prepared as an account of work sponsored by an agency of the United States Government. Neither the United States Government nor any agency thereof, nor any of their employees, makes any warranty, express or implied, or assumes any legal liability or responsjbility for the accuracy, completeness, or usefulness of any information, apparatus, product, or process disclosed, or represents that its use would not infringe privately owned rights. Reference herein to any specific commercial product, process, or service by trade name, trademark, manufacturer, or otherwise does not necessarily constitute or imply its endorsement, recommendation, or favoring by the United States Government or any agency thereof. The views and opinions of authors expressed herein do not necessarily state or reflect those of the United States Government or any agency thereof.

\section{HIGH ENERGY IONIZATION IN LIQUIDS - THE FREE ION YIELD}

\author{
Richard Holroyd \\ Department of Chemistry \\ Brookhaven National Laboratory \\ Upton, NY 11973
}

\section{INTRODUC̣TION}

Ionizations can be produced in liquids in many ways, for example by exposure to high energy radiation (fast electrons or alphas) or by interaction with ultraviolet photons of sufficient energy. The passage of a high energy electron results in well separated clusters, each containing a few ionizations or in the case of alpha particles a dense column of ionization. Absorption of ultraviolet light creates a single ion-electron pair. In any case only a fraction of the ion-electron pairs formed initially separate to become free; in most liquids recombination within the track or cluster dominates. The yield of ion electron pairs that separate and become free depends on several factors including the molecular structure of the solvent, the density of ionization along the track, the liquid density, the applied field and the temperature ${ }^{1}$.

First we will consider the situation of a single isolated ion-electron pair. The electron is formed with initial kinetic energy which allows it to travel some distance away from its geminate cation. The electron may lose energy to vibrational modes but a significant fraction of the scparation occurs while the electron has subvibrational (near thermal) energy. When the electron finally thermalizes or localizes it is within the Coulombic field of its parent cation and the two ions constitute a geminate pair. The free ion yield is determined by the fraction of geminate pairs which diffuse out to form free ions as against those that recombine to form excited states. The probability of escape of an isolated geminate pair, where the thermalization distance is $r$, is given at zero field by:

$$
\mathrm{P}_{\mathrm{esc}}^{0}(\mathrm{r})=\exp (-\mathrm{rd} \mathrm{r})
$$

where $r_{c}$ is the Onsager radius, which is that distance at which the Coulombic energy drops to the thermal energy $(k T)$; i.e., $r_{c}=e^{2} / \varepsilon k T$. Generally $P_{e s c}$ is a function of the electric field, the temperature, the dielectric constant as well as the thermalization distance, $r$. Thus the yield of free ions or number of ion pairs that separate per $100 \mathrm{eV}$ absorbed is: 
The initial yield of ionization, $\mathrm{G}_{\mathrm{tot}}$, is not known exactly for molecular liquids. A value equal to the gas phase yield $(100 / \mathrm{W})$ is often assumed, where $\mathrm{W}$ is the number of eV required to form an ion pair. For neopentane the extrapolated yield at high field is 4.0 per $100 \mathrm{eV}{ }^{2}$ Thus the free ion yield depends on $G_{t o t}$ and $P(r, E)$, the escape probability, which depends strongly on the thermalization distance. Note that a distribution of thermalization distances $D(r)$ is assumed, usually Gaussian or exponential. Although irradiation by electrons of $\mathrm{NieV}$ energy leads to clusters of ionization, the above formalism is often used to analyze the results.

In this talk we consider some recent experimental results which show how $\mathrm{G}_{\mathrm{fi}}$ depends on conditions. The results provide further insight into the factors which determine the free ion yield.

\section{EXPERIMENTAL METYIODS}

The methods employed for measurements of the free ion yield differ in the way in which charge is collected. All methods require a measurement of the dose absorbed in order to calculate $\mathrm{G}_{\mathrm{fi}}$. In the steady irradiation method the current is measured with an electric field (E) applied to a sample exposed to a beam of ionizing radiation. The ion yield increases with field, as discussed below, but the results can be extrapolated to zero field to get $G_{f}$, the zero field yield. When this method is used care must be taken to avoid volume recombination of ions. The efficiency of ion collection for continuous irradiation is given by 3 :

$$
\begin{aligned}
\therefore \quad \text { Eff. } & =\frac{2}{1+\left(1+z^{2}\right)^{1 / 2}} \\
\text { where } z^{2} & =\frac{2 \mathrm{ed}^{2} \mathrm{DR}}{3 \varepsilon_{\mathrm{o}} \varepsilon \mathrm{E}^{2}}\left(\frac{1}{\mu_{ \pm}}+\frac{1}{\mu}\right)
\end{aligned}
$$

Thus the efficiency decreases as $\mathrm{d}$, the distance between plates, or DR, the dose rate increases. The efficiency increases as $\mathrm{E}$, the field, or $\mu$, the mobility, increase. The efficiency of ion collection is improved by working at low dose rates which means measurement of low currents (generally less than $10^{-10} \mathrm{~A}$ ). ${ }^{3}$

Other methods involve collection of charge following a pulse of irradiation. Ion yields for many liquids were determined by the clearing field method. 4,5 In this technique a field large enough to avoid volume recombination is applied quickly after a short pulse in order to collect all the ions. This method gives $\mathrm{G}_{\mathrm{fi}}^{\mathrm{O}}$ directly since there is no field present during the pulse. Another method involves measuring the charge following a pulse of irradiation where a steady electric field is applied. ${ }^{6}$ Here, as in the steady state method, the field enhances the ion yield and the observed charges are extrapolated to zero field to obtain $G_{f i}^{0}$. The efficiency of ion collection is given for the pulsed case by: 7

$$
\begin{gathered}
\text { Eff }=\frac{1}{U} \ln (1+U) \\
\text { where } U=\operatorname{ed~} N_{\alpha} / \varepsilon_{0} \varepsilon E .
\end{gathered}
$$

The efficiency will decrease as $\mathrm{d}$ and $\mathrm{N}_{0}$, the initial concentration of ions, increase and will increase as $E$ increases. A recent study 7 shows that the different methods give similar results for hexane, where $G_{f i}^{0}=0.13 \pm .01$ ions $/ 100 \mathrm{eV}$.

Another method of determining the ion yield is based on the electron yield from passage of a single ionizing particle. The concentration of charged species is much lower, a charge 
sensitive amplifier is employed. Here the efficiency is a function of the concentration of electron trapping impurities, or specifically $R$ which is equal to $\tau / t_{d}$, where $\tau$ is the electron lifetime and $\tau_{d}$ is the drift time. The efficiency of collection is: 8

$$
\mathrm{Eff}=2 \mathrm{R}+2 \mathrm{R}^{2}[\exp (-1 / \mathrm{R})-1]
$$

The efficiency increases with $R$ and is not very good unless $R \gg 1$.

\section{MOLECULAR STRUCTURE EFFECTS}

Examination of the data shown in Table 1 indicates that free ion yields for liquids are dependent on molecular structure. $G_{f i}^{0}$ ranges from a low value of 0.01 for ethane to values nearly a hundred-fold larger for the tetramethyl compounds. The reason for this large range of values is not entirely clear. $G_{f \mathfrak{f}}$ depends on the initial separation of electron and ion, which in turn depends on the rate of energy loss of the electron and on the smoothness of the potential seen by epithermal electrons slowing down in the conduction band. Electrons must lose energy rapidly in ethylene and acetylene. The thermalization distance in these liquids is quite short $(<4 \mathrm{~nm}),{ }^{\mathbb{1}}$ but the process responsible is as yet unidentified.

Free ion yields are also quite low for compounds with conjugated double bonds indicating an efficient energy loss mechanism is present. Here temporary anion formation is likely to be involved. 10 Electron attachment to 1,3-butadiene and benzene has been shown to occur in liquids at high pressure ${ }^{11}$ and in glasses a: low temperature.12,13 Such temporary anions provide a mechanism for energy loss by the electron which limits its separation distance from the positive ion.

Table 1 - Free Ion Yields ${ }^{a}$

\begin{tabular}{llll}
\hline Liquids & $\begin{array}{l}\text { Temp } \\
\text { oK }\end{array}$ & $\begin{array}{l}\text { Electrons } \\
\mathrm{G}_{\mathrm{fi}} \mathrm{o}\end{array}$ & $\begin{array}{l}\text { Alphas } \\
\mathrm{G}_{\mathrm{fi}} @ \mathrm{E}=10 \mathrm{kV} / \mathrm{cm}\end{array}$ \\
\hline Ethene & 170 & 0.01 & \\
Acetylene & 198 & 0.02 & \\
1,3-Butadiene & 269 & 0.04 & \\
Benzene & 296 & 0.05 & \\
trans -2-Butene & 293 & 0.08 & \\
n-Hexane & 296 & 0.13 & $0.017^{\mathrm{d}}$ \\
n-Tetradecane & 296 & 0.12 & \\
Carbon tetrachloride & 296 & 0.10 & \\
Germanium tetrachloride & 296 & 0.13 & \\
Tetraethylsilane & 295 & $0.18 \mathrm{~b}$ & \\
Tetraethylgermane & 295 & $0.18^{\mathrm{b}}$ & \\
Tetraethylstannane & 295 & $0.20^{\mathrm{b}}$ & \\
2,2,4-Trimethylpentane & 295 & 0.33 & $0.018^{\mathrm{e}}$ \\
Tetramethylsilane & 295 & $0.65^{\mathrm{c}}$ & $0.029 \mathrm{e}$ \\
Tetramethylgermane & 295 & $0.63^{\mathrm{c}}$ & $0.029 \mathrm{f}$ \\
Tetramethylstannane & 295 & $0.64^{\mathrm{c}}$ & \\
2,2,4,4-Tetramethylpentane & 295 & 0.83 & $0.025^{\mathrm{e}}$ \\
Neopentane & 296 & 1.10 & $0.036^{\mathrm{e}}$ \\
\hline
\end{tabular}

a) Data from Ref 9 unless indicated otherwise

b) Ref 14

c) $\operatorname{Ref} 15$

d) $\operatorname{Ref} 16$

e) $\operatorname{Ref} 17$

f) $\operatorname{Ref} 18$

For tetraalkyl compounds containing a central $\mathrm{Si}, \mathrm{Ge}$ or $\mathrm{Sn}$ atom the free ion yields span quite a range. ${ }^{14,15}$ The yields depend strongly on the nature of the alkyl group. The yields 
are comparable, $\mathrm{G}_{\mathrm{fi}}^{0}=0.64 \pm 0.01$, for the three tetramethyl-compounds in Table 1 . The ion yields are much less for the tetraethyl-compounds, $G_{f i}^{0}=0.19 \pm .01$, and even lower for compounds with larger side groups. For these liquids the density of the $\mathrm{Sn}$-containing liquid is about twice the density of the corresponding Si-containing liquid showing that the density alone does not determine the yield. Rather the thermalization distance is dependent on the nature of the alkyl group.

The smoothness of the potential seen by the epithermal electron is important. Evidently symmetrical struc cures with many methyl groups provide the smoothest potential leading to the largest range. The lower yield for the tetraethyl compounds indicates the important role of methyl groups. Although the tetrachloro-compounds are symmetrical, the yields are lower because electron attachment occurs.

The product be also appear to be an important parameter. For the tetramethyl compounds discussed earlier $G_{f i}$ is nearly the same and be is also the same. Recently from an analysis of free ion yield data for 52 liquids at room temperature it was shown ${ }^{19}$ that $\log$ $\mathrm{G}_{\mathrm{fi}}^{\mathrm{O}}$ is linearly dependent on $(\mathrm{b} \varepsilon)^{-1}$, a dependence ex

Implicit in the above discussion is the assumption that thermalization precedes electron trapping. However electrons may be trapped before complete thermalization as has been suggested. 20 This provides another mechanism limiting the separation distance. It also provides a rationalization for the known correlation of thermal electron mobility with mean thermalization distance (see Ref 1) since the magnitude of the mobility is related to the probability of trapping. 10

\section{FIELD AND TEMPERATURE EFFECTS}

The probability of separation of an electron-ion pair in an electric field as developed by Onsager ${ }^{21}$ is of the form:

$$
P(E, r)=\exp (-r d r)[1+F(E, r)]
$$

When averaged over all angles between the direction of the external field and the electron-ion axis, $\mathrm{F}(\mathrm{E}, \mathrm{r})$ becomes: 22

$$
F(E, r)=\exp \left(-U_{0}\right) \sum_{n=1}^{\infty} \frac{U_{0}^{n}}{(n+1) !} \sum_{j=0}^{n-1} \frac{(n-j)\left(r_{d} / r\right)(j+1)}{(j+1) !}
$$

where $\mathrm{U}_{\mathrm{o}}=\mathrm{eEr} / \mathrm{k}_{\mathrm{B}} \mathrm{T}$. At low field (small $\mathrm{U}_{\mathrm{O}}$ ):

$$
F(E, r)=e^{3} E / 2 \varepsilon\left(k_{b} T\right)^{2} \text { or }\left(9.68 / \varepsilon T^{2}\right) E
$$

and the ratio of slope-to-intercept of plots of ion yields versus field should be $9.68 / \varepsilon T^{2}$ $\mathrm{cm} / \mathrm{V}$. Typically the values of slope-to-intercept are close to this value for liquids where $\mathrm{G}_{\mathrm{fi}}$ is small. See for example the data for $n$-hexane in figure 1 where $G_{f_{i}}$ is near 0.1 per $100 \mathrm{eV}$. For higher fields and for liquids of higher free ion yield, where the dependence of yield on field becomes nonlinear at a relatively low field, the full equation (4) is required to adequately describe the datal. 
Fig. 1 Plot of $\mathrm{G}_{\mathrm{fi}}$ versus field for $\mathrm{n}$-hexane at indicated pressures (in bar).

Free ion yields increase with increasing temperature. This is associated with the temperature dependence of the Onsager relation, equation (3). For single ion pairs created by photoioni zation the temperature dependence is given well by equation ( 3$)^{23}$. For high energy radiacion the mean thermalization distance, $b$, increases with increasing temperature because the density(d) of the liquid is decreasing. Then the product bd is considered an important parameter. This parameter is nearly constant for $n$-pentane and benzene over a large range of temperatures. However, bd varies considerably for 2,2,4-trimethylpentane and neopentane. 5 For such liquids made up of spherelike molecules with many methyl groups, temperature studies show that the product bd increases with temperature and goes through a maximum at a temperature just below the critical temperature. Examples of liquids exhibiting such behavior are 2,2,4,4-tetramethylpentane ${ }^{24}$ and neopentane (see Fig 2). The mobility of thermal electrons also shows a maximum at nearly the same temperature for these compounds. This indicates the scattering of thermal electrons is similar to that of epitherma! electrons and that the potential seen by both types of electron is relatively smooth at the maximum.

It is important to realize that determinations of the mean thermalization range usually are done by assuming some distribution of ranges, $D(r)$, typically Gaussian, and fitting the data to equation 2 for an assumed value of $G_{t o t}$. However $G_{t o t}$ is not known for most liquids and the value of the range parameter derived changes when $G_{t o t}$ is changed. Also a Gaussian distribution may not be the best description of reality. Recently it was shiwn for the tetra-alkyl liquids 25 that if the form of $D(r)$ is the same in all liquids then $G_{t o t}$ must vary from liquid to liquid, or conversely that if $G_{t o t}$ is the same then the form of $D(r)$ changes. 
Fig. 2 Plot of bd vs density for neopentane; and plot of nue vs density for neopentane (Ref 1 ).

\section{TRACK EFFECTS}

The discussion so far has dealt with ion yields for liquids exposed to high energy electrons (in the MeV range). Such energetic electrons are minimum-ionizing and the clusters of ionization are well separated. As the energy of the electron decreases the clusters will occur closer together because the rate of energy loss increases with decreasing energy. For a $10 \mathrm{keV}$ electron the initial positions of the clusters will be, on average, $10 \mathrm{~nm}$ apart. Because of interactions between clusters the situation is best described as a track. The free ion yield will be less than for minimum ionizing radiation because of the enhanced possibility of ion-electron recombination in such a track.

One way to study how ion yields vary with electron energy is with $\mathrm{X}$-rays since absorption of an X-ray of energy in the 2 to $30 \mathrm{keV}$ range by a hydrocarbon results in a photoelectron of comparable energy. The new synchrotron radiation sources provide tunable $\mathrm{X}$-rays in this range. Dosimetry is readily done with an ion chamber inserted in the X-ray beam ahead of the cell containing the liquid sample. 
Fig. 3 Variation of zero field free ion yield with $X$-ray energy for $2,2,4,4$-tetramethylpentane, $O$ 2,2,4-trimethylpentane and $\bullet$-hexane.

The variation of the free ion yield with $\mathrm{X}$-ray energy is shown in figure 3 for three hydrocarbons. 26,27 For all three the yield decreases with decreasing $X$-ray energy to minimum values around $2 \mathrm{keV}$. The magnitude of the effect depends on the hydrocarbon. For $n$-hexane $G_{\mathrm{fi}}^{\mathrm{o}}$ decreases a factor of about two but for 2,2,4,4-tetramethylpentane the yield decreases a factor of about eight. Qualitatively this effect can be understood as an effect of the rate of energy loss, $\mathrm{d} E / \mathrm{dx}$, of the photo-electron which increases with decreasing $\mathrm{X}$-ray energy. This results in a higher density of ionization which increases the probability of electrons combining with other ions in the tracks.

A comparison of experimental data with a theoretical predication 28 is shown in Fig 4 . A computer was used to simulate diffusion and drift of ions and electrons in tracks, taking the nonhomogeneous kinetics of ion recombination into account. The theory predicts $\mathrm{G}_{\mathrm{fi}}^{\mathrm{O}}$ decreases with decreasing energy for both $n$-hexane and 2,2,4-trimethylpentane in agreement with experiment. The theory also predicts the yields increase again at very low energy. 
Fig 4 Dependence of $G_{f i}^{0}$ on electron energy: experimental from ref 25 and 26 for $O$ $2,2,4$-trimethylpentane and $n$-hexane lines are theory, ref $28, G_{10 t}=5$ and $b=10.6 \mathrm{~nm}$ for 2,2,4-trimethylpentane and $6.8 \mathrm{~nm}$ for $\mathrm{n}$-hexane. $\therefore$

There are a few studies of ion yields in liquid hydrocarbons exposed to very densely ionizing particles like alpha particles. ${ }^{16-18}$ Results for some liquids at $10 \mathrm{kV} / \mathrm{cm}$ are given in Table 1. In contrast to high energy electrons there is less of a molecular structure effect for alphas; the yields are within a factor of two for those liquids that have been studied. The field dependence of the ion yields for alpha radiation of hydrocarbons can be empirically expressed by an equation of the form:

$$
\mathrm{G}_{\mathrm{fi}}=\mathrm{G}_{\mathrm{fi}}^{\mathrm{o}}+\alpha \mathrm{E}^{\mathrm{n}}
$$

where $\mathrm{n}$ is 0.7 to 0.8 and $\alpha$ depends on the liquid. The value of the intercept, $\mathrm{G}_{\mathrm{fj}}^{0}$ is very small, about 0.005 per $100 \mathrm{eV}$ for alphas. A yield of zero is expected for the cylindrical geometry of a track in contrast to the spherical geometry of isolated ion pairs where $G_{f i}^{\circ}$ is finite (see equation 1).

The theoretical understanding of alpha ray yields dates back to the analysis of Jaffé29 published in 1913. A cylindrical distribution of positive and negative ions was assumed to be formed initially. The calculation of the ion yield under an applied field requires consideration of the diffusion as well as the recombination of the ions. To solve the differential equation Jaffé first ignored the recombination, solved the simplified equation, and added the recombination later. His well known equation predicts the dependence of the free ion field to be given by

$$
1 / \mathrm{G}_{\mathrm{fi}}=1 / \mathrm{G}_{\mathrm{fi}}^{\infty}+\mathrm{B} \frac{\mathrm{d} E}{\mathrm{dx}} \frac{\mathrm{S}(\mathrm{z})}{\mathrm{E}}
$$

where $S(z)$ is a function of $E, r$, and temperature (see Ref 26). Thus the yield should increase with field and decrease as $\mathrm{d} E / \mathrm{dx}$ (the rate of energy loss) increases. Figure 5 shows some results for exposure of hydrocarbons to electrons (from X-rays) 26,27 . The data points are $\mathrm{G}_{\mathrm{fi}}$ for an applied field of $5 \mathrm{kV} / \mathrm{cm}$. The dashed lines are best fits of equation 7 to the 
$\mathrm{X}$-ray ciata. The values of $\mathrm{G}^{\infty}$ used are $0.16,0.25$ and 0.42 per $100 \mathrm{eV}$ for $\mathrm{n}$-hexane, 2,2,4-trimethylpentane and 2,2,4,4-tetramethylpentane, respectively. The slopes, B S(z)/E, are $6.30,4.70$ and $3.58 \times 10^{-8} \mathrm{~cm}$ for $\mathrm{n}$-hexane, 2,2,4-trimethylpentane and 2,2,4,4-tetramethylpentane, respectively. The lines have been extrapolated to higher values of $\mathrm{d} E / \mathrm{dx}$. The values predicted for alphas in this way for $\mathrm{d} E / \mathrm{dx}=10 \mathrm{x} 10^{8} \mathrm{eV} / \mathrm{cm}$, are higher (by $60 \%$ or less) than the experimental points.

Fig. 5 Dependence of ion yields at $5 \mathrm{kV} / \mathrm{cm}$ on $\mathrm{dE} / \mathrm{dX}$ for $\Delta 2,2,4,4$-tetramethylpentane, 2,2,4-trimethylpentane, and $O \mathrm{n}$-hexane. Dashed lines eq 7-see text.

Further progress in understanding alpha tracks must take into account the detailed structure of the track. For example the electrons initially are expected to have a wider distribution than the positive ions. The difference in distribution will produce an internal electric field. Consequently, recombination will be very important. In fact Kramers' theoretical analysis ${ }^{30}$ neglected diffusion initally and he solved the equation including recombination first and added diffusion later. For hydrocarbons the probability that electrons will escape the main track of an alpha particle is very low. Electrons which thermalize some distance from the track are more likely to contribute to the observed yield. These may be electrons on the tail of the distribution or may be ionizations caused by delta rays which are secondary electrons with enough energy to leave the track. Escape of ion pairs formed in the delta ray track is much more likely.

\section{DENSITY EFFECTS}

To determine how the free ion yield varies with liquid density, measurements are made as a function of pressure. In this way the temperature can be held constant. Applications of pressure to $3 \mathrm{kbar}$ increases the density of a hydrocarbon by about $20 \%$. Measurements of this sort are illustrated for $n$-hexane in figure $1 . G_{\mathrm{fi}}^{0}$ decreases with increasing pressure and at $3 \mathrm{kbar}$ is $55 \%$ of its value at 1 bar. Over this range the density increases by $20 \%$. Thus the mean thermalization distan ie is experted to decrease with increasing pressure because of 
the increase in number of scattering centers. Analysis of the data in figure 1 indicates the product bd decreases by $10 \%$ over this pressure range. ${ }^{31}$.

\section{ACKNOWLEDGEMENT}

This research was carried out at Brookhaven National Laboratory under contract DE-AC02-76CH00016 with the U.S. Department of Energy and supported by its Division of Chemical Sciences, Office of Basic Energy Sciences.

\section{REFERENCES}

1. G.R. Freeman, "Ionization and charge separation in irradiated materials " in "Kinetics of nonhomogeneous processes" G.R. Freeman, ed. J. Wiley and Sons NY. (1987).

2. W.F. Schmidt, Radiation-induced conductivity and ion yields in neopentane at high electric fields, Radiation Research 42:73 (1970).

3. W.F. Schmidt, R.A. Holroyd, Ion mobilities and yields in X-irradiated polydimethylsiloxane oils, Radiat. Phys. Chem. 39:349 (1992).

4. W.F. Schmidt, A.O. Allen, Ionization of liquids by radiation: measurements by a clearing field, Science 160:301 (1968).

5. W.F. Schmidt, A.O. Allen, Free ion yields in sundry irradiated liquids, J. Chem. Phys. 52:2345 (1970).

6. G. Ramanan, N. Gee, G.R. Freeman, Electron energy lnss in fluids: thermalization distances in liquid and gaseous sulfur hexafluoride. Can. J. Phys. 68:925 (1990).

7. N. Gee, C. Senanayake, G. R. Freeman, Electron mobility, free ion yields, and electron thermalization distances in n-alkane liquids: effect of chain length, J. Chem. Phys. 89:3710 (1989).

8. R. Holroyd, Effects of radiation damage to TMP, TMS and liquid argon solutions, in Radiation Effects at the SSC, M.G. D. Gilchriese, ed. SSC-SR-1035 p339 (1988).

9. A.O. Allen, Yields of free ions formed in liquids by radiation, NSRDS-NBS-57 (1976).

10. J-P. Dodelet, K. Shinsaka, G.R. Freeman, Electron mobilities in liquid olefins: structure effects, J. Chem. Phys. 59:1293 (1973).

11. R.A. Holroyd, Electron reactions in nonpolar liquids - pressure effects, proceedings-this ASI.

12. T. Shida, W.H. Hamill, Molecular Ions VI. Electronic absorption and electron paramagnetic resonance spectra of molecular ions of conjugated dienes and allyl radicals, J. Am. Chem. Soc. 88:5371 (1966).

13. T Shida, S. Iwata, Electronic spectra of Ion radicals and their molecular orbital interpretation III aromatic hydrocarbons, J. Am. Chem. Soc. 95:3473 (1973).

14. 14.R. Holroyd, Free ion yields in liquids-molecular structure effects, in "Proceedings International Conference on Liquid Radiation Detectors: Their Fundamental Properties and Applications", Waseda Univ. Tokyo, Japan p24 (1992).

15. R. A. Holroyd, S. Geer, F. Ptohos, Free-ion yields for several silicon-, germanium, and tin-containing liquids and their mixtures, Phys. Rev. B 43:9003 (1991).

16. M. Chybicki, lonization currents induced by alpha radiation in liquid hexane and heptane, Acta Phys. Pol, 30:927 (1966).

17. R. C. Munoz, J. B. Cumming, R. A. Holroyd, Ionization of liquid hydrocarbons and tetramethylsilane by ${ }^{241}$ Am alpha particles, J. Chem. Phys. 85:1104 (1986). 
18. R. A. Holroyd, in "SSC Detector R and D a: $2: \mathrm{L}^{\mathrm{L}}$ ", B. Yu and V. Radeka, eds, BNL 52244 p23 (1990).

19. J. -P. Jay-Gerin, T. Goulet, I. Billard, On the correlation between electron mobility, free-ion yield, and electron thermalization distance in nonpolar dielectric liquids, Can J. Chem. 71:287 (1993).

20. B.S. Yakovlev, Low energy electron localization in hydrocarbon glasses. Method of photoassisted ion pair separation, Radiat. Phys. Chem 40:37 (1992).

21. L. Onsager, Initial recombination of ions, Phys. Rev, 54:554 (1938).

22. J. Terlecki, J. Fiutak, Onsager's recombination theory applied to liquid alkanes, Int. J. Radiation Phys. and Chem. 4:469 (1972).

23. R.A. Holroyd, R.L. Russell, Solvent and temperature effects in the photoionization of tetramethyl-p-phenylenediamine, J. Phys. Chem. 78:2128 (1974).

24. T.G. Ryan, G.R. Freeman, Electron mobilities and ranges in methyl substituted pentanes through the liquid and critical regions, J. Chem. Phys., 68:5144 (1978).

25. S. Geer, R.A. Holroyd, Electron thermalization lengths and total initial ionization yields in tetra-alkyl liquids, Phys. Rev. B. 46:5043 (1992).

26. R.A. Holroyd and T.K. Sham, Ion yields in hydrocarbon liquids exposed to X-rays of 5-30 keV Energy, J. Phys. Chem. 89:2909 (1985).

27. R.A. Holroyd, T.K. Sham, B.-X. Yang and X.-H. Feng, Free Ion yields exposed to synchrotron X-rays, J. Phys. Chem. 96:7438 (1992).

28. W. M. Bartczak, A. Hummel, Computer simulation study of spatial distribution of the ions and electrons in tracks of high energy electrons and the effect on the charge recombination, J. Phys. Chem. 97:1253 (1993).

29. G. Jaffé, Zur theorie der ionisation in kolonnen, Annalen der Physik 42:303 (1913).

30. H.A. Kramers, On a modification of Jaffé's theory of column - ionization, Physica $18: 665$ (1952).

31. R.A. Holroyd and E. Stradowska, unpublished. 
miv $1+1 .>0$ (GFIHEX.WKI)

also rtared on 1230 d

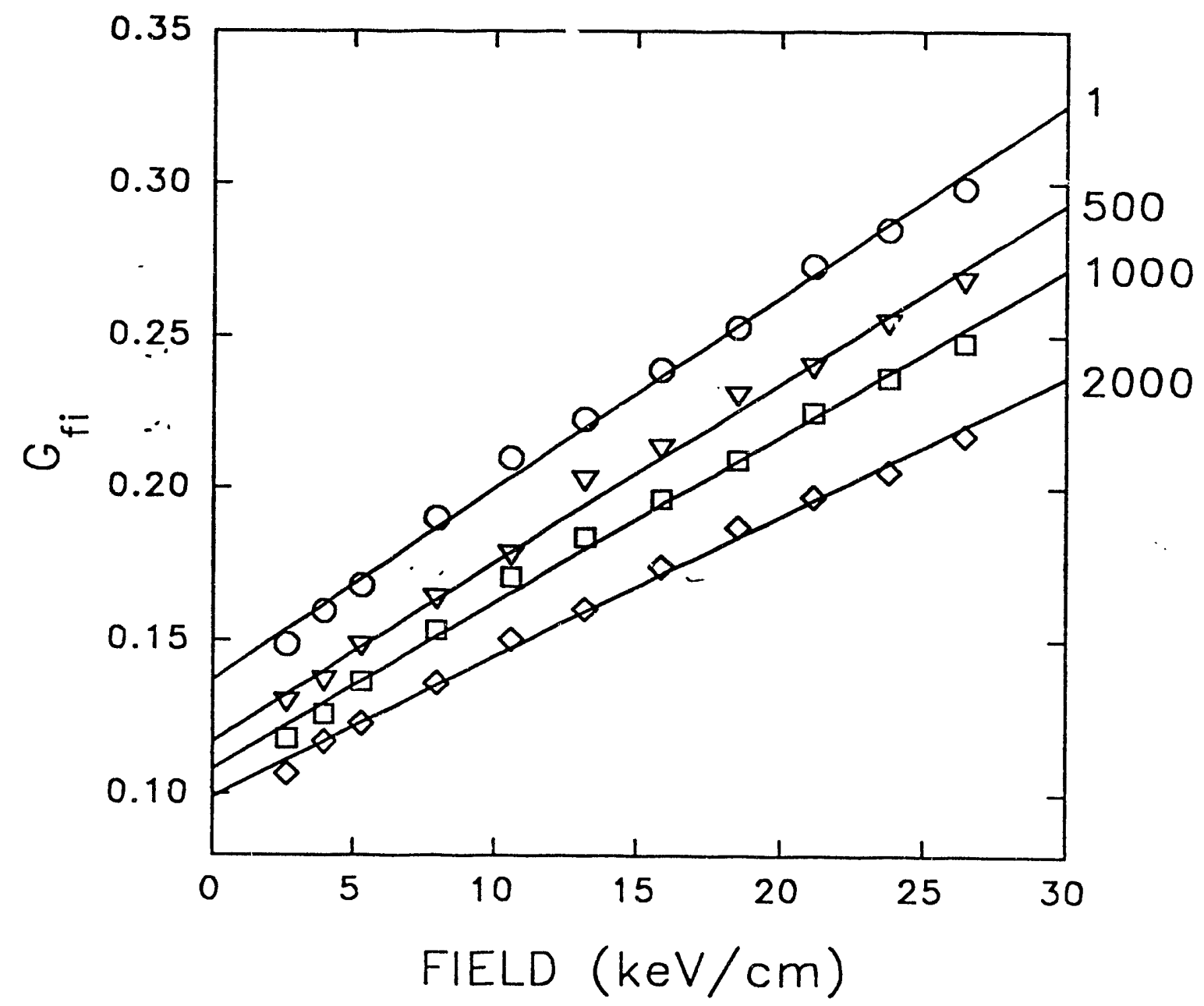




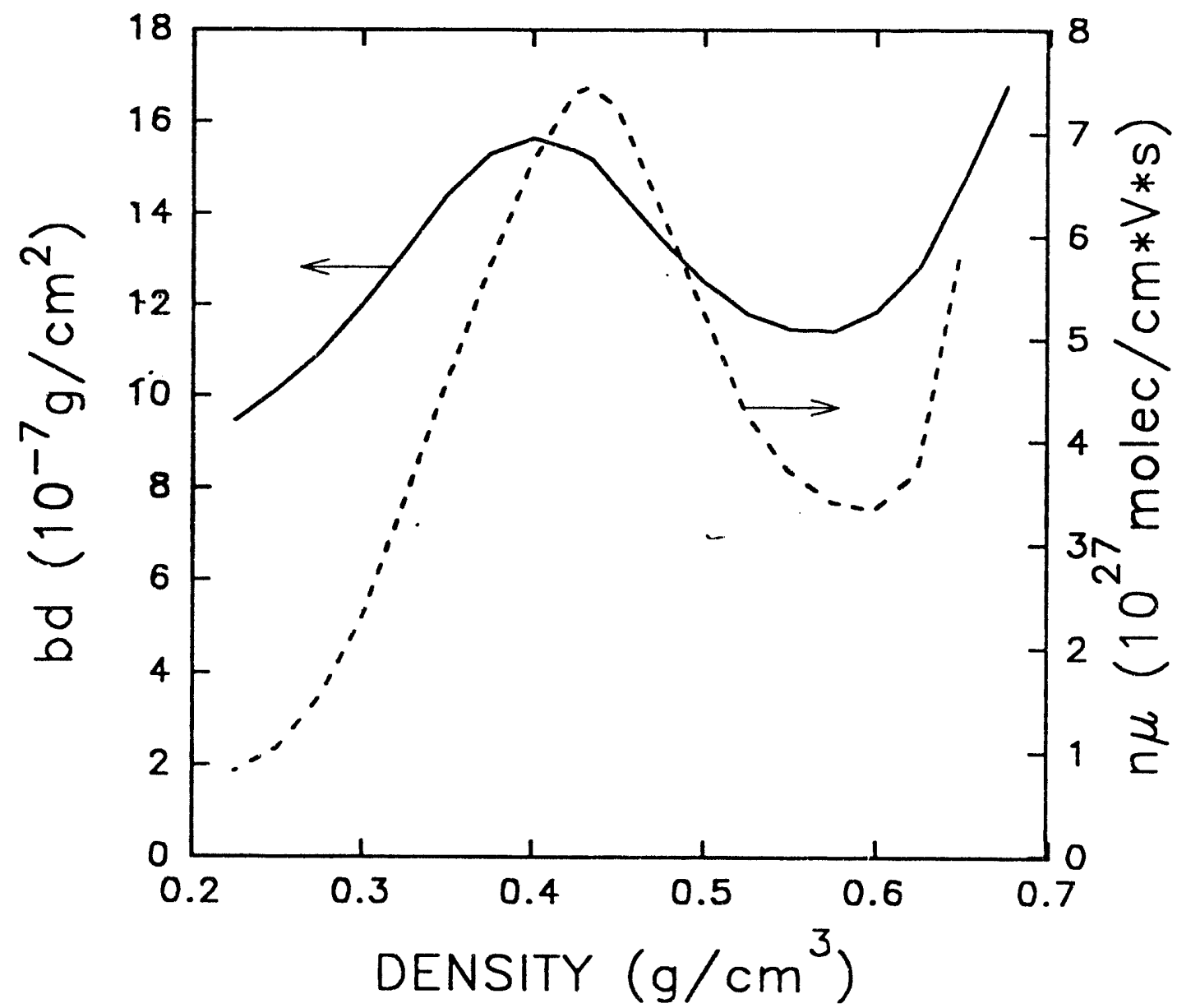

$\therefore F / G 2$ 


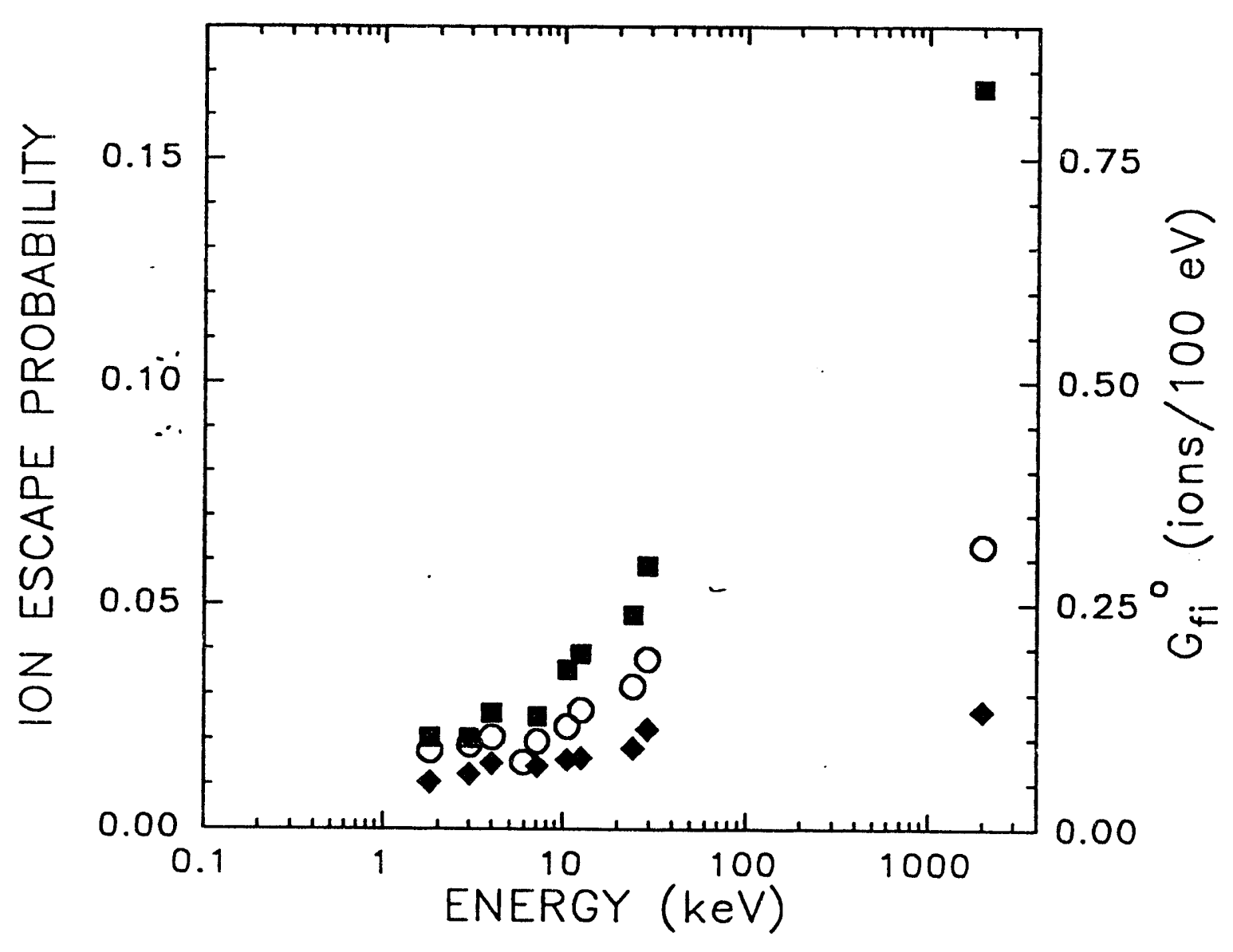




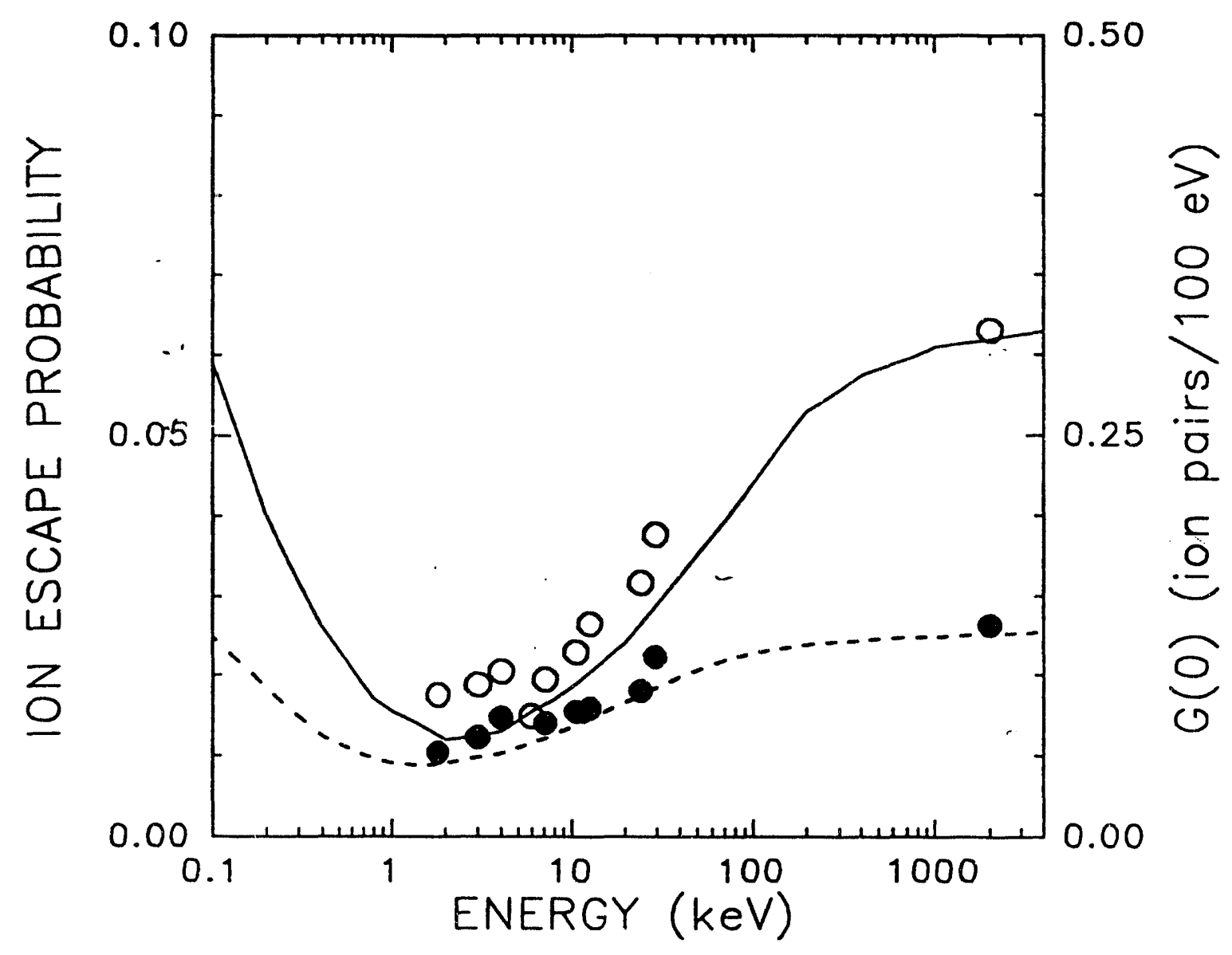

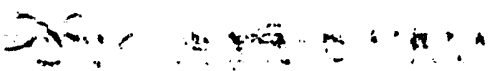




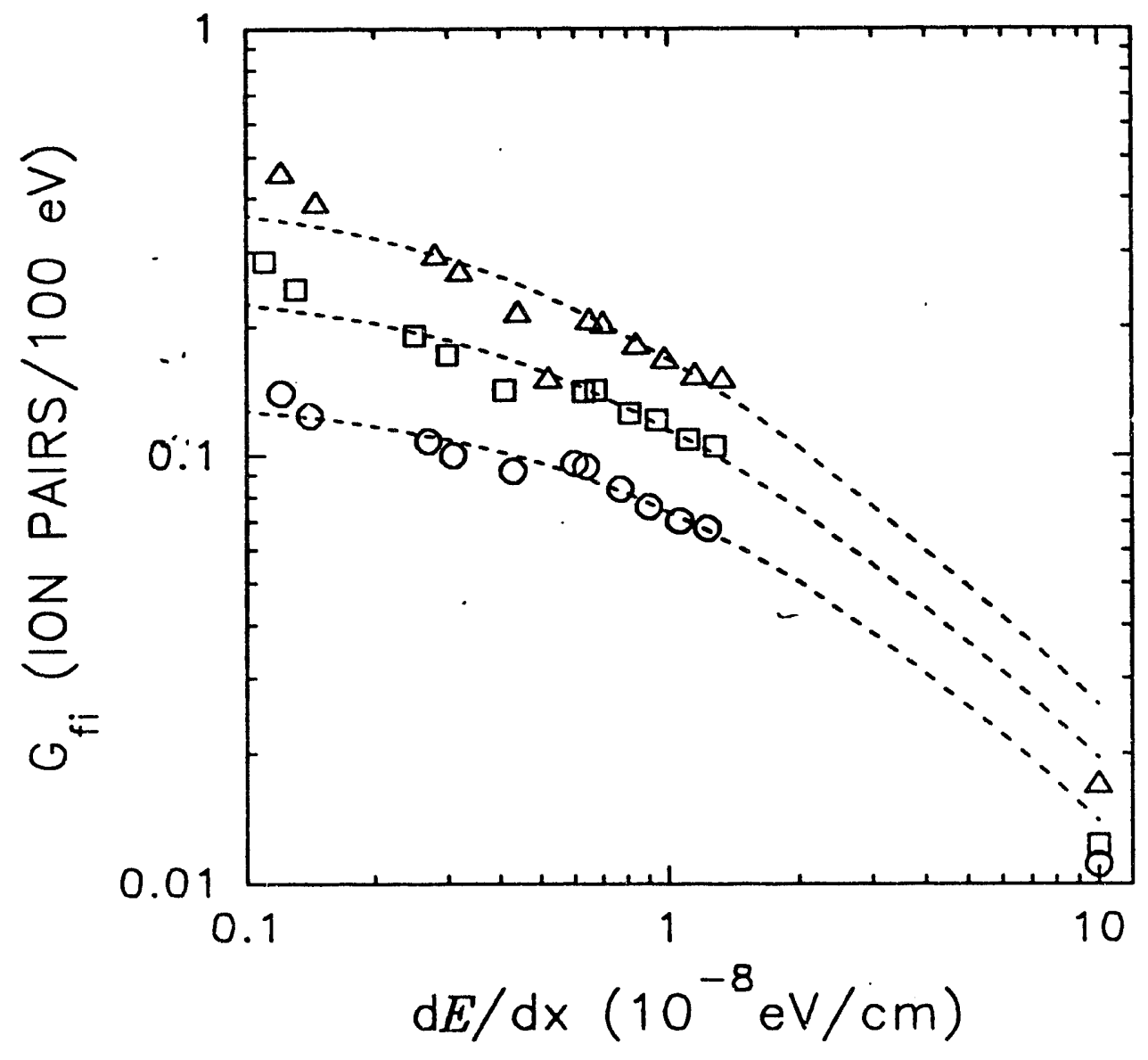



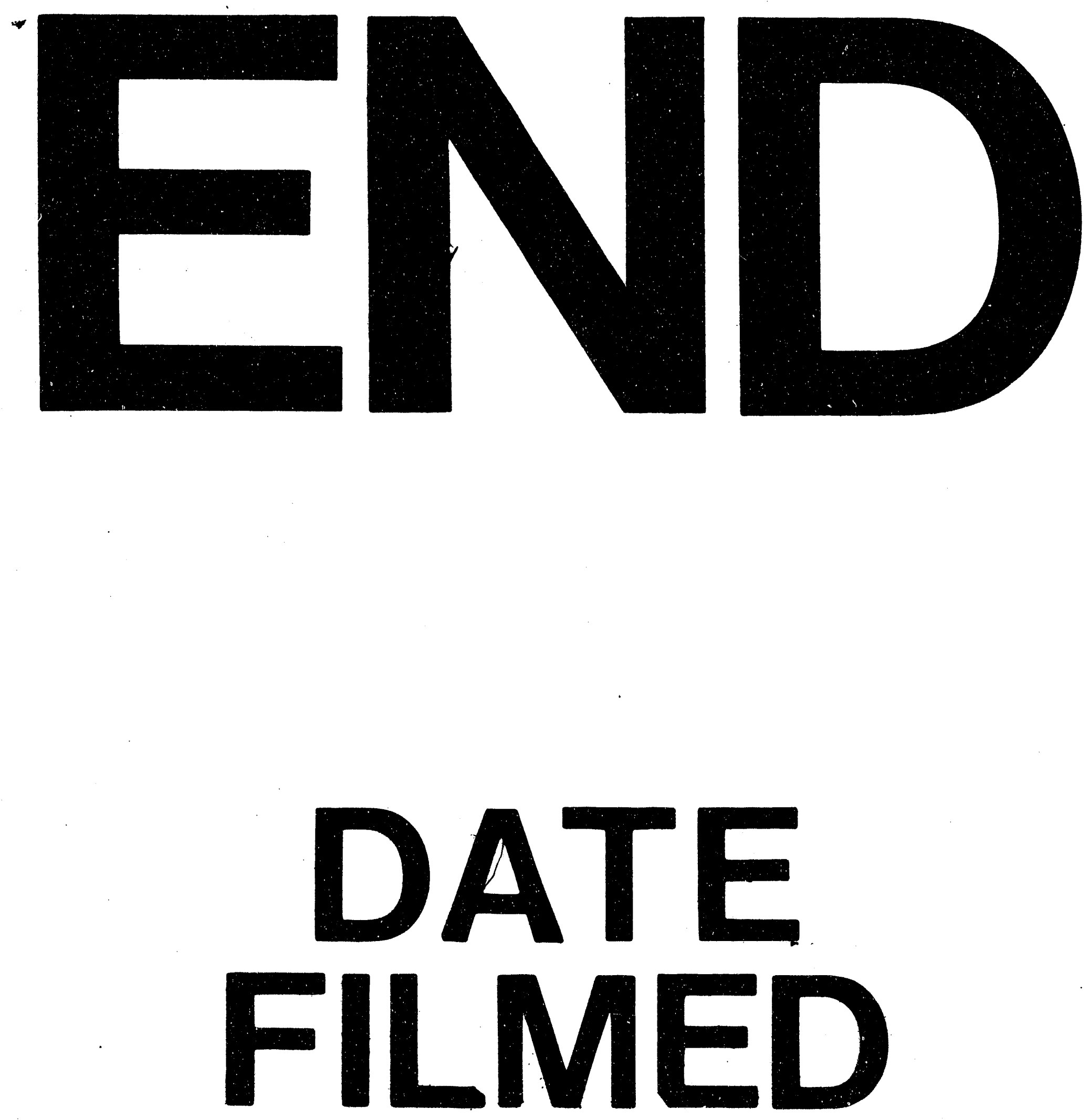

1

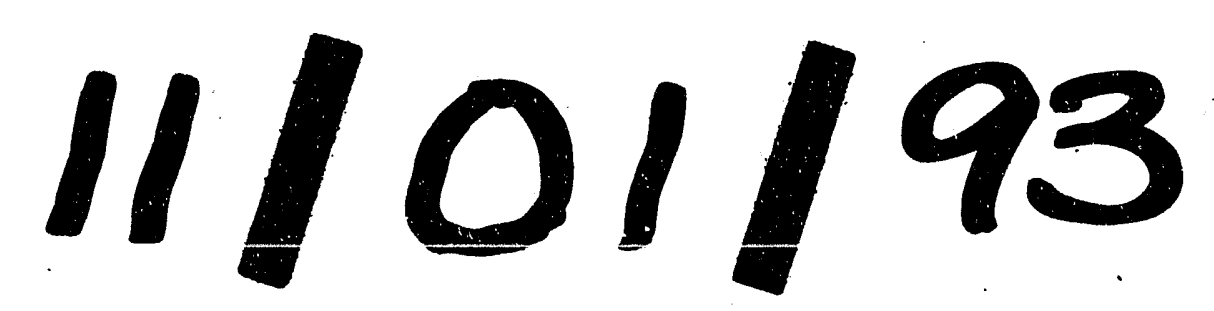


